\title{
COVID-19 disease transmission model considering direct and indirect transmission
}

\author{
Dipo Aldila* \\ Department of Mathematics, Indonesia University, Depok 16424, Indonesia
}

\begin{abstract}
A mathematical model for understanding the COVID-19 transmission mechanism proposed in this article considering two important factors: the path of transmission (direct-indirect) and human awareness. Mathematical model constructed using a four-dimensional ordinary differential equation. We find that the Covid-19 free state is locally asymptotically stable if the basic reproduction number is less than one, and unstable otherwise. Unique endemic states occur when the basic reproduction number is larger than one. From sensitivity analysis on the basic reproduction number, we find that the media campaign succeeds in suppressing the endemicity of COVID-19. Some numerical experiments conducted to show the dynamic of our model respect to the variation of parameters value.
\end{abstract}

\section{Introduction}

Formerly come from Wuhan, China at the end of 2019, COVID-19 keep spread through worldwide and threat human respiratory system [1]. Until June 22, 2020, more than nine million people worldwide have been infected by COVID-19, where the USA is the highest contributor of this number of the recorded case [2].

Covid-19 believed to be transmitted originally from animal to human. However, it is more dominant that the infection was coming from direct contact of human-to-human, or from contact with contaminated surfaces which contain droplets from infected individual [3]. Several interventions have been conducted by policymakers in many countries, such as physical distancing, use of medical mask, rapid assessment to trace infected individuals, use of disinfectant, and many more [3].

Mathematical models have been conducted by many authors to understand how OCVID19 spread among the population [5-13]. In this study, continue our previous study on the COVID-19 transmission model [7]. The idea is coming from the fact that media campaigns become one of the most important interventions by policymakers to increase human awareness of the danger of COVID-19. This media campaign will separate the population into aware and non-aware subpopulations. The question that needs to be addressed then: How well is the media campaign could reduce the spread of COVID-19? What is the most influential parameter in determining the existence of COVID-19? Hence, this article will be addressed the above questions based on mathematical model analysis.

\footnotetext{
* Corresponding author : aldiladipo@sci.ui.ac.id
} 
Our article organized as follow. A mathematical model formulation is given in Section 2 and followed with mathematical analysis regarding the steady-state, basic reproduction number, and local stability criteria in Section 3. Furthermore, we conduct some numerical examples in Section 4. Finally, some conclusion is given in Section 5.

\section{Model Formulation}

Here in this article, we construct our model based on our previous result in [7]. The modification lies in additional awareness of the population due to the media campaign by the government. The purpose of this media campaign is to develop an awareness of susceptible populations to practice a healthy lifestyle against COVID-19 viruses, such as the use of medical mask and routine to wash hands after contact with the suspicious object that might bring virus on the surface.

Hence, let the human population divided into three subpopulations, namely susceptible unaware $S_{u}$, susceptible aware $S_{a}$, and infected population $I$. We also include one additional compartment which consider the free viruses in the environment, namely $V$.

Susceptible unaware population increases due to newborn $\Lambda$, drop out from unaware population due to vanishment of awareness $u_{2}$, and recovered from Covid-19 $\alpha$ with a proportion of $p$. On the other hand, it decreases due to "direct" transmission from an infected population with successful infection rate $\beta_{1}$, and from "indirect" transmission from contact with free viruses with successful contact rate $\beta_{2}$, and transition to the aware population due to media campaign $u_{1}$.

Susceptible aware population increases due to transition from an unaware population with the rate of $u_{1}$, and recovery from the disease at a constant rate of $\alpha$ with proportion of $(1-p)$. Furthermore, it decreases due to "direct" and "indirect" transmission with infection probability $\beta_{1}$ and $\beta_{2}$, and transition to unaware population due to vanishment of awareness with rate of $u_{2}$. Since we assume that aware population implements healthy life-style, the transmission probability reduced by $q$ for the direct transmission, and by $r$ for indirect transmission.

Infected population increases due to infection from an aware and unaware population, and decreases due to recovery from COVID-19. On the other hand, the free Virus increases from infected population which produces viruses from sneezed and decreases due to natural inactivated rate $\mu_{v}$.

Based on the above assumptions, the COVID-19 transmission model considers directindirect transmission and aware population describe by the following four-dimensional ordinary differential equation.

$$
\begin{aligned}
& \frac{d S_{u}}{d t}=\Lambda-\beta_{1} S_{u} I-\beta_{2} S_{u} V-\mu_{h} S_{u}+p \alpha I-u_{1} S_{u}+u_{2} S_{a}, \\
& \frac{d S_{a}}{d t}=u_{1} S_{u}-u_{2} S_{a}-q \beta_{1} S_{a} I-r \beta_{2} S_{a} V-\mu_{h} S_{a}+(1-p) \alpha I, \\
& \frac{d I}{d t}=\beta_{1} S_{u} I+\beta_{2} S_{u} V+q \beta_{1} S_{a} I+r \beta_{2} S_{a} V-\mu_{h} I-\alpha I, \\
& \frac{d V}{d t}=\xi I-\mu_{v} V
\end{aligned}
$$

where $\mu_{h}$ is the natural death rate. This system is supplemented by non-negative initial condition:

$$
S_{u}(0)=S_{u 0} \geq 0, S_{a}(0)=S_{a 0} \geq 0, I(0)=I_{0} \geq 0, V(0)=V_{0} \geq 0 .
$$


It is easy to see that since $\frac{d\left(S_{u}+S_{a}+I\right)}{d t}=\frac{d N}{d t}=\Lambda-\mu_{h} N$, we have that $\lim _{t \rightarrow \infty} N=\frac{\Lambda}{\mu_{h}}$. Using the vector field approach, we can see that $S_{u}, S_{a}, I$, and $V$ always has an inward gradient whenever it approaches 0 . Hence, we conclude that our model in (1) is biologically well posed.

\section{Model analysis}

\subsection{The Covid-19 free state and the basic reproduction number}

System (1) has a trivial equilibrium when an infected population does not exist in the population. We call this equilibrium as the COVID-19 free state, which given by

$$
E_{0}=\left(S_{u}, S_{a}, I, V\right)=\left(\frac{\Lambda\left(\mathrm{u}_{1}+u_{2}\right)}{\mu_{h}\left(\mu_{h}+u_{1}+u_{2}\right)}, \frac{\Lambda \mathrm{u}_{1}}{\mu_{h}\left(\mu_{h}+u_{1}+u_{2}\right)}, 0,0\right) .
$$

Next, we calculate the basic reproduction number using the next-generation matrix approach [14]. System (1) has a basic reproduction number given by

$$
R_{0}=\frac{\Lambda\left(\beta_{1} \mu_{v}\left[\mu_{h}+u_{2}+q u_{1}\right]+\xi \beta_{2}\left[\mu_{h}+u_{2}+r u_{1}\right]\right)}{\mu_{h} \mu_{v}\left(\mu_{h}+u_{1}+u_{2}\right)\left(\alpha+\mu_{h}\right)} .
$$

This basic reproduction number could be re-written based on the source of infection (direct and indirect transmission) as follows :

$$
R_{0}=\frac{\Lambda\left(\beta_{1}\left[\mu_{h}+q u_{1}+u_{2}\right]\right)}{\underbrace{\mu_{h}\left(\mu_{h}+u_{1}+u_{2}\right)\left(\alpha+\mu_{h}\right)}_{\text {direct transmission }}}+\frac{\Lambda\left(\xi \beta_{2}\left[\mu_{h}+r u_{1}+u_{2}\right]\right)}{\underbrace{\mu_{h} \mu_{v}\left(\mu_{h}+u_{1}+u_{2}\right)\left(\alpha+\mu_{h}\right)}_{\text {indirect transmission }}}
$$

From the above expression, it can be seen that the component of direct transmission depends on the number of maximum populations $\frac{\Lambda}{\mu_{h}}$ multiplied with direct transmission rate $\beta_{1}$. Hence, we conclude that a larger number of the population will increase direct transmission. On the other hand, from indirect transmission component, it appears that the infection depends on the multiplication between transmission rate $\beta_{2}$, a total of human population $\frac{\Lambda}{\mu_{h}}$ and a maximum number of free viruses $\frac{\xi}{\mu_{v}}$. Further discussion on $R_{0}$ will be discussed in the later section of this article.

In many epidemiological models, basic reproduction number holds an important role in determining the stability of the disease-free state [15-21]. It is common to find that the disease-free state is locally asymptotically stable if $R_{0}<1$ and unstable otherwise. Similarly, using the linearization approach, we have the following theorem.

Theorem 1. The Covid-19 free state $E_{0}$ of system (1) is locally asymptotically stable (LAS) if $R_{0}<1$, and unstable otherwise.

Proof. Linearize system (4) at $\Gamma_{0}$ yield

$$
J_{0}=\left[\begin{array}{ll}
J_{1,1} & J_{1,2} \\
J_{2,1} & J_{2,2}
\end{array}\right]
$$

where $J_{1,1}=\left[\begin{array}{cc}-\left(\mu_{h}+u_{1}\right) & u_{2} \\ u_{1} & -\left(\mu_{h}-u_{2}\right)\end{array}\right], J_{1,2}=\left[\begin{array}{cc}\alpha p-\frac{\Lambda \beta_{1}\left(\mu_{h}+u_{2}\right)}{\mu_{h}\left(\mu_{h}+u_{1}+u_{2}\right)} & -\frac{\Lambda \beta_{2}\left(\mu_{h}+u_{2}\right)}{\mu_{h}\left(\mu_{h}+u_{1}+u_{2}\right)} \\ (1-p) \alpha-\frac{\Lambda \mathrm{q} \beta_{1} u_{1}}{\mu_{h}\left(\mu_{h}+u_{1}+u_{2}\right)} & -\frac{\Lambda \mathrm{r} \beta_{2} u_{1}}{\mu_{h}\left(\mu_{h}+u_{1}+u_{2}\right)}\end{array}\right], J_{2,1}=\left[\begin{array}{ll}0 & 0 \\ 0 & 0\end{array}\right]$ , and $J_{2,2}=\left[\begin{array}{cc}-\left(\alpha+\mu_{h}\right)+\frac{\beta_{1} \Lambda\left(\mu_{h}+q u_{1}+u_{2}\right)}{\mu_{h}\left(\mu_{h}+u_{1}+u_{2}\right)} & \frac{\beta_{2} \Lambda\left(\mu_{h}+q u_{1}+u_{2}\right)}{\mu_{h}\left(\mu_{h}+u_{1}+u_{2}\right)} \\ \xi & -\mu_{v}\end{array}\right] . J_{0}$ has two negative eigenvalues, i.e $\lambda_{1}=$ $-\mu_{h}$ and $\lambda_{2}=-\left(\mu_{h}+u_{1}+u_{2}\right)$. The other two eigenvalues are coming from the root of the following polynomial:

$$
a_{2} \lambda^{2}+a_{1} \lambda+a_{0}=0
$$

where $a_{2}=-\mu_{h}\left(\mu_{h}+u_{1}+u_{2}\right), \quad a_{1}=\Lambda \beta_{1}\left(\mu_{h}+q u_{1}+u_{2}\right)-\mu_{h}\left(\mu_{h}+u_{1}+u_{2}\right)(\alpha+$ $\left.\mu_{h}+\mu_{v}\right)$, and $a_{0}=\mu_{h} \mu_{v}\left(\mu_{h}+u_{1}+u_{2}\right)\left(\alpha+\mu_{h}\right)\left(R_{0}-1\right)$. 
To guarantee this polynomial has a negative real part for all it roots, it should be that $\lambda_{3} \lambda_{4}=\frac{a_{0}}{a_{2}}>0$ and $\lambda_{3}+\lambda_{4}=-\frac{a_{1}}{a_{2}}<0$. It is easy to see that $\lambda_{3} \lambda_{4}=\frac{a_{0}}{a_{2}}>0 \Leftrightarrow R_{0}<1$. On the other hand, $\lambda_{3}+\lambda_{4}=-\frac{a_{1}}{a_{2}}<0 \Leftrightarrow \frac{\Lambda \beta_{1}\left(\mu_{h}+q u_{1}+u_{2}\right)}{\mu_{h}\left(\mu_{h}+u_{1}+u_{2}\right)\left(\alpha+\mu_{h}+\mu_{v}\right)}<1$. Since

$$
\frac{\Lambda \beta_{1}\left(\mu_{h}+q u_{1}+u_{2}\right)}{\mu_{h}\left(\mu_{h}+u_{1}+u_{2}\right)\left(\alpha+\mu_{h}+\mu_{v}\right)}<R_{0}<1
$$

Then we have that $E_{0}$ is LAS if $R_{0}<1$, and unstable otherwise. Hence, the proof is completed.

From the form of the basic reproduction number, when $\beta_{v}=0$, which indicate that we neglect the indirect transmission, we have that

Hence, we have the following remark.

$$
R_{0}^{*}=\frac{\Lambda\left(\beta_{1}\left[\mu_{h}+q u_{1}+u_{2}\right]\right)}{\mu_{h}\left(\mu_{h}+u_{1}+u_{2}\right)\left(\alpha+\mu_{h}\right)}<R_{0} .
$$

Remark 1. Indirect transmission increases the standard basic reproduction number. The increases of this basic reproduction number highly dependent on the number of human population and the free viruses on the environment. Hence, reducing the number of viruses could using any intervention to kill the free viruses could be considered as an alternative option to eradicate COVID-19 transmission.

\subsection{The Covid-19 endemic state}

System (1) has a nontrivial state, which called the endemic state. This state is given by

$$
E_{1}=\left(S_{u}, S_{a}, I, V\right)=\left(S_{u}^{*}, \frac{u_{1} S_{u}^{*}+\alpha I^{*}(1-p)}{u_{2}+\mu_{h}+\beta_{2} r V^{*}+\beta_{1} q I^{*}}, I^{*}, \frac{\xi I^{*}}{\mu_{v}}\right),
$$

where $S_{u}^{*}$ has a complex expression to be written in this article, while $I^{*}$ taken from the positive roots of the following polynomial

where

$$
b_{2} I^{2}+b_{1} I+b_{0}=0
$$

$$
\begin{aligned}
& b_{2}=-\mu_{h}\left(\xi \beta_{2}+\beta_{1} \mu_{v}\right)\left(q \beta_{1} \mu_{v}+r \xi \beta_{2}\right) \\
& b_{1}=\left(\xi \beta_{2}+\beta_{1} \mu_{v}\right)\left(q \beta_{1} \mu_{v}+r \xi \beta_{2}\right) \Lambda-\mu_{h} \mu_{v}\left(\alpha \xi \beta_{2}(1-p)+\alpha \beta_{1} \mu_{v}(1-p) . .\right. \\
& \quad+\alpha p q \beta_{1} \mu_{v}+\alpha p r \xi \beta_{2}+q \beta_{1} \mu_{h} \mu_{v}+q \beta_{1} \mu_{h} u_{1}+r \xi \beta_{2} \mu_{h}+r \xi \beta_{2} u_{1}+\cdots \\
& \left.\quad+\xi \beta_{2} \mu_{h} \xi \beta_{2} u_{2}+\beta_{1} \mu_{h} \mu_{v}+\beta_{1} \mu_{v} u_{2}\right) \\
& b_{0}=\mu_{v} \mu_{h} \mu_{v}\left(\mu_{h}+u_{1}+u_{2}\right)\left(\alpha+\mu_{h}\right)\left(R_{0}-1\right) .
\end{aligned}
$$

Hence, system (1) has:

a) Unique endemic equilibrium if $R_{0}>1$, or $R_{0}<1, b_{1}>0$ and $b_{1}^{2}-4 b_{0} b_{2}=0$, or $R_{0}=1$ and $b_{1}<0$.

b) Two endemic equilibrium if $R_{0}<1, b_{1}>0$ and $b_{1}^{2}-4 b_{0} b_{2}>0$.

c) No endemic equilibrium otherwise.

Using the linearization of system (1) at $E_{1}$, the Jacobian matrix is given by

$$
K_{1}=\left[\begin{array}{ll}
K_{1,1} & K_{1,2} \\
K_{2,1} & K_{2,2}
\end{array}\right]
$$

where $\quad K_{1,1}=\left[\begin{array}{cc}-\beta_{1} I^{*}-V^{*} \beta_{2}-\left(\mu_{h}+u_{1}\right) & u_{2} \\ u_{1} & -I^{*} q \beta_{1}-V^{*} r \beta_{2}-\left(\mu_{h}+u_{2}\right)\end{array}\right], \quad K_{1,2}=$ $\left[\begin{array}{cc}\alpha p-S_{u}^{*} \beta_{1} & -S_{u}^{*} \beta_{2} \\ -q \beta_{1} S_{a}^{*}+(1-p) \alpha & -r \beta_{2} S_{a}^{*}\end{array}\right], \quad K_{2,1}=\left[\begin{array}{cc}\beta_{1} I^{*}+\beta_{2} V^{*} & q \beta_{1} I^{*}+r \beta_{2} V^{*} \\ 0 & 0\end{array}\right]$, and $K_{2,2}=$ $\left[\begin{array}{cc}q \beta_{1} I^{*}+\beta_{1} S_{u}^{*}-\alpha-\mu_{h} & r \beta_{2} S_{a}^{*}+S_{u}^{*} \beta_{2} \\ \xi & -\mu_{v}\end{array}\right]$. The characteristic polynomial of $K_{1}$ is given by 


$$
\sum_{i=0}^{4} a_{i} \lambda^{i}=0
$$

where $a_{i}$ for $i=0,1, \ldots, 4$ is a long expression to be shown in this article. Hence, based on Routh-Hurwitz stability criteria, $E_{1}$ will locally stable if

$$
a_{0}>0, a_{3}>0, a_{3} a_{2}-a_{4} a_{1}>0,\left(a_{3} a_{2}-a_{4} a_{1}\right) a_{1}-a_{3}^{2} a_{0}>0 \text {. }
$$

We close this section with the following remark.

Remark 2. The endemic of COVID-19 will always be established whenever the basic reproduction number is larger than one. Based on the form of the basic reproduction number, we notice that indirect transmission is equally important for the focus of the eradication program compared with an intervention that focuses on minimizing the direct transmission.

\section{Numerical experiments}

We focus on two questions in this section. The first question is to what is the most sensitive parameter that determines $R_{0}$ ? It is very important to notice what controllable parameter that can be used to modify the size of $R_{0}$ most efficiently. The next question is, how is the longtime behavior of our model respect to the change of parameter value? Hence, we conduct an autonomous simulation to answer this question.

\subsection{Sensitivity analysis}

We have observed from the previous section that the basic reproduction number holds an essential role in the transmission of COVID-19. Here, we perform the local sensitivity analysis [22] to understand the robustness of the basic reproduction number to the parameter value. The normalized sensitivity analysis of variable $c$ respect to $R_{0}$, denoted by $\Gamma_{c}^{R_{0}}$, defined as

$$
\Gamma_{c}^{R_{0}}=\frac{\partial R_{0}}{\partial c} \times \frac{c}{R_{0}} .
$$

For an example, since $\frac{\partial R_{0}}{\partial \beta_{1}}=\frac{\Lambda\left(\mathrm{qu}_{1}+\mu_{h}+u_{2}\right)}{\mu_{h}\left(\mu_{h}+u_{1}+u_{2}\right)\left(\alpha+\mu_{h}\right)}$, then we have

$$
\begin{aligned}
\Gamma_{\beta_{1}}^{R_{0}} & =\frac{\Lambda\left(\mathrm{qu}_{1}+\mu_{h}+u_{2}\right)}{\mu_{h}\left(\mu_{h}+u_{1}+u_{2}\right)\left(\alpha+\mu_{h}\right)} \times \frac{\beta_{1} \mu_{h} \mu_{v}\left(\mu_{h}+u_{1}+u_{2}\right)\left(\alpha+\mu_{h}\right)}{\Lambda\left(\beta_{1} \mu_{v}\left[\mu_{h}+u_{2}+q u_{1}\right]+\xi \beta_{2}\left[\mu_{h}+u_{2}+r u_{1}\right]\right)} \\
& =\frac{\left(\mathrm{qu}_{1}+\mu_{h}+u_{2}\right) \beta_{1} \mu_{v}}{\left(\beta_{1} \mu_{v}\left[\mu_{h}+u_{2}+q u_{1}\right]+\xi \beta_{2}\left[\mu_{h}+u_{2}+r u_{1}\right]\right)} .
\end{aligned}
$$

Using the following parameters

$$
\begin{gathered}
\Lambda=\frac{10^{6}}{65 \times 365}, \mu_{h}=\frac{1}{65 \times 365}, \mu_{v}=\frac{1}{3}, \beta_{1}=0.2 \times 10^{-7}, \beta_{2}=0.2 \times 10^{-11}, \\
u_{1}=0.1, u_{2}=0.05, \alpha=\frac{1}{30}, q=0.1, r=0.1, \xi=10^{4},
\end{gathered}
$$

we have that $\Gamma_{\beta_{1}}^{R_{0}}=0.625$. It means that increasing $\beta_{1}$ for $10 \%$ will increase $R_{0}$ to $6.25 \%$. Using the same parameters value, we have that $\Gamma_{u_{1}}^{R_{0}}=-0.499$, which means that increasing $u_{1}$ for $10 \%$ will reduce $R_{0}$ for $4.99 \%$. With similar approach, the sensitivity index of $\mu_{h}, \beta_{2}, u_{2}, \mu_{v} q, r, \xi$ and $\alpha$ is $-1.00084,0.375,0.4995,-0.375,0.104,0.0625,0.375$, and 0.9987 , respectively. We have shown this phenomenon for all parameters in Fig.1. It can be seen that $u_{1}$ and $u_{2}$ are promising to reduce $R_{0}$ since the sensitivity index is negative and positive, respectively. Fig. 2 shows the contour plot of the basic reproduction number respect 
to $u_{1}$ and $u_{2}$. It can be seen clearly that for increasing value of $u_{1}$ will reduce $R_{0}$. On the other hand, $u_{2}$ should be reduced to reduce $R_{0}$.

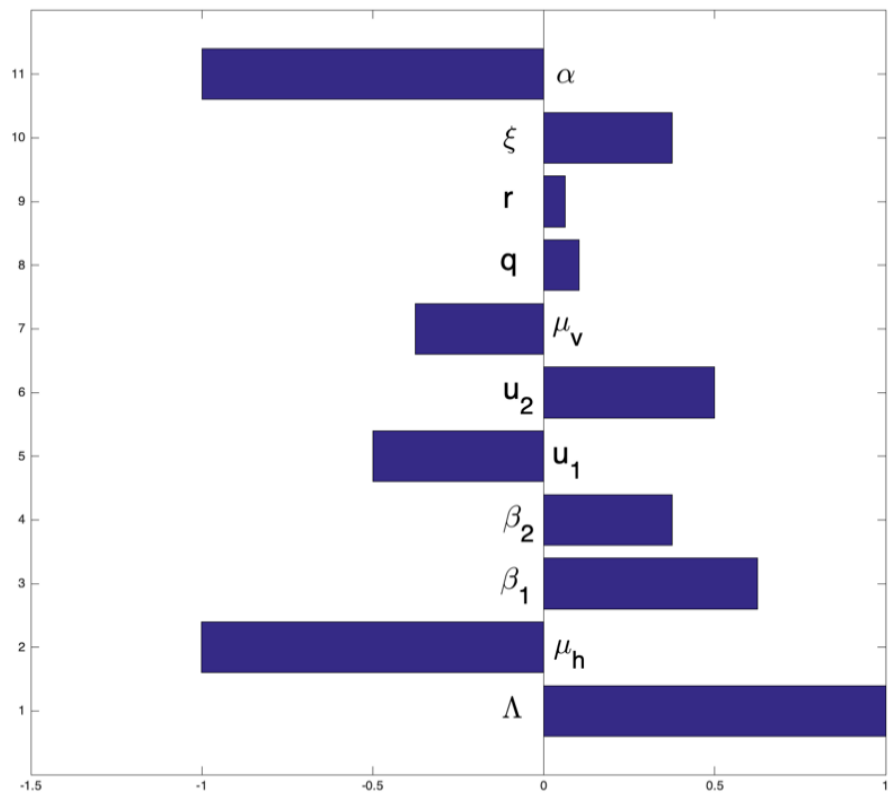

Fig. 1. Tornado plot for the sensitivity of $R_{0}$ respect to parameters of model (1).

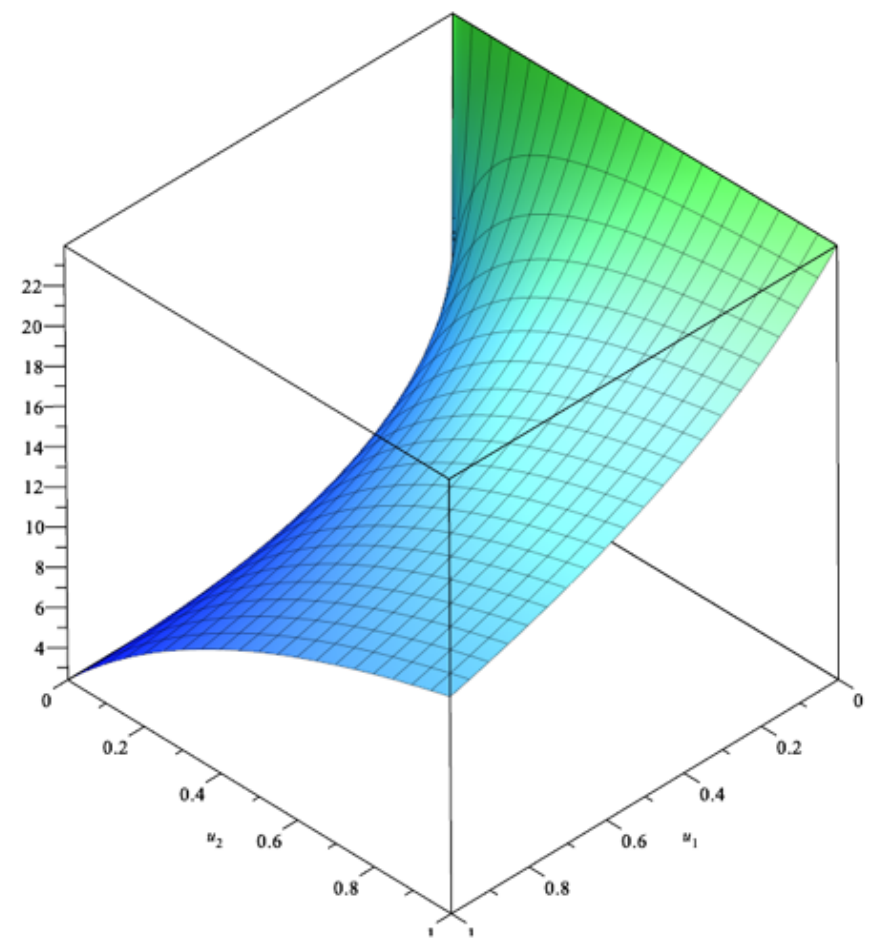

Fig. 2. Contour plot of dependency of $R_{0}$ to $u_{1}$ and $u_{2}$. 


\subsection{Numerical simulation of the Autonomous system}

As previously mentioned, our goal in this section is to visualize the effect of parameter value to the dynamical solution of our proposed COVID-19 model in (1). For the first example, we will show how our model changed respect to the change of media campaign to transfer unaware subpopulation to the aware population $\left(u_{1}\right)$. To conduct the simulation, we use the following parameters

$$
\begin{aligned}
\Lambda=\frac{10^{6}}{65 \times 365}, \mu_{h} & =\frac{1}{65 \times 365}, \mu_{v}=\frac{1}{3}, \beta_{1}=0.2 \times 10^{-7}, \beta_{2}=0.2 \times 10^{-11}, \\
u_{2} & =0.05, \alpha=\frac{1}{30}, q=0.1, r=0.1, \xi=10^{4},
\end{aligned}
$$

while $u_{1}$ are $0,0.025,0.05$ and 0.4 which give us the basic reproduction number 4.79, 3.35, 2.63 and 0.96, respectively. The result is shown in Fig. 3. Based on Theorem 1, we have that only when $u_{1}=0.4$ will give us a stable COVID-19 free state, while the other value of $u_{1}$ will make the COVID-19 exist in the population.

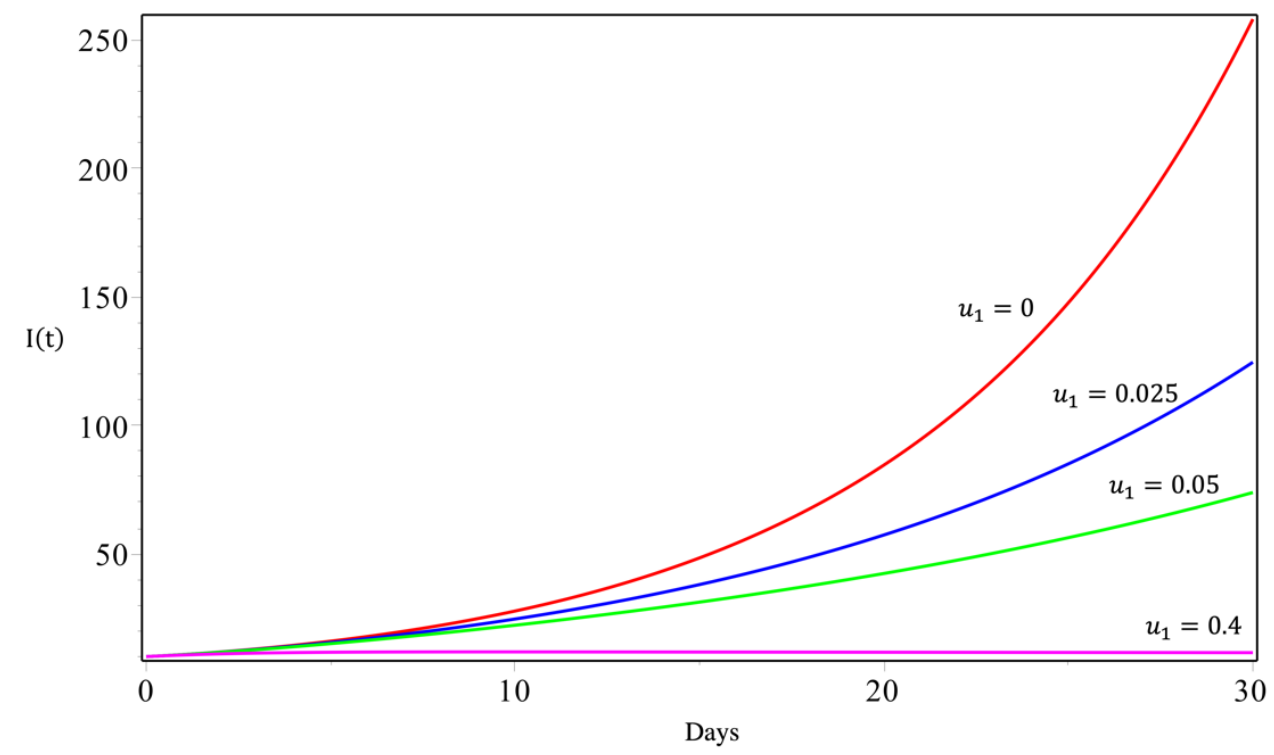

Fig. 3. Solution of trajectories of infected population of model (1) for various value of $u_{1}$.

The next simulation conducted using the following parameters value

$$
\begin{gathered}
\Lambda=\frac{10^{6}}{65 \times 365}, \mu_{h}=\frac{1}{65 \times 365}, \mu_{v}=\frac{1}{3}, \beta_{1}=0.2 \times 10^{-7}, \beta_{2}=0.2 \times 10^{-11}, \\
u_{1}=0.1, \alpha=\frac{1}{30}, q=0.1, r=0.1, \xi=10^{4},
\end{gathered}
$$

while $u_{2}$ are $0.1,0.05,0.025$ and 0.01 which gave us the basic reproduction number 2.63, 1.91, 1.34 and 0.87, respectively. The result is shown in Fig. 4. Again, based on Theorem 1, we have that only when $u_{2}=0.01$ will give us a stable COVID-19 free state, while the other value of $u_{2}$ will make the COVID-19 exist in the population. Based on these simulations, we can conclude that it needs a proper balance of $u_{1}$ and $u_{2}$ to achieve a condition where COVID-19 no longer exists in the population. It shows us how important is the media campaign to educate the population about the danger of COVID-19. This intervention 
expected to increase the awareness and make the aware population reduce the possibility of infection by COVID-19, whether from direct or from indirect transmission pathway.

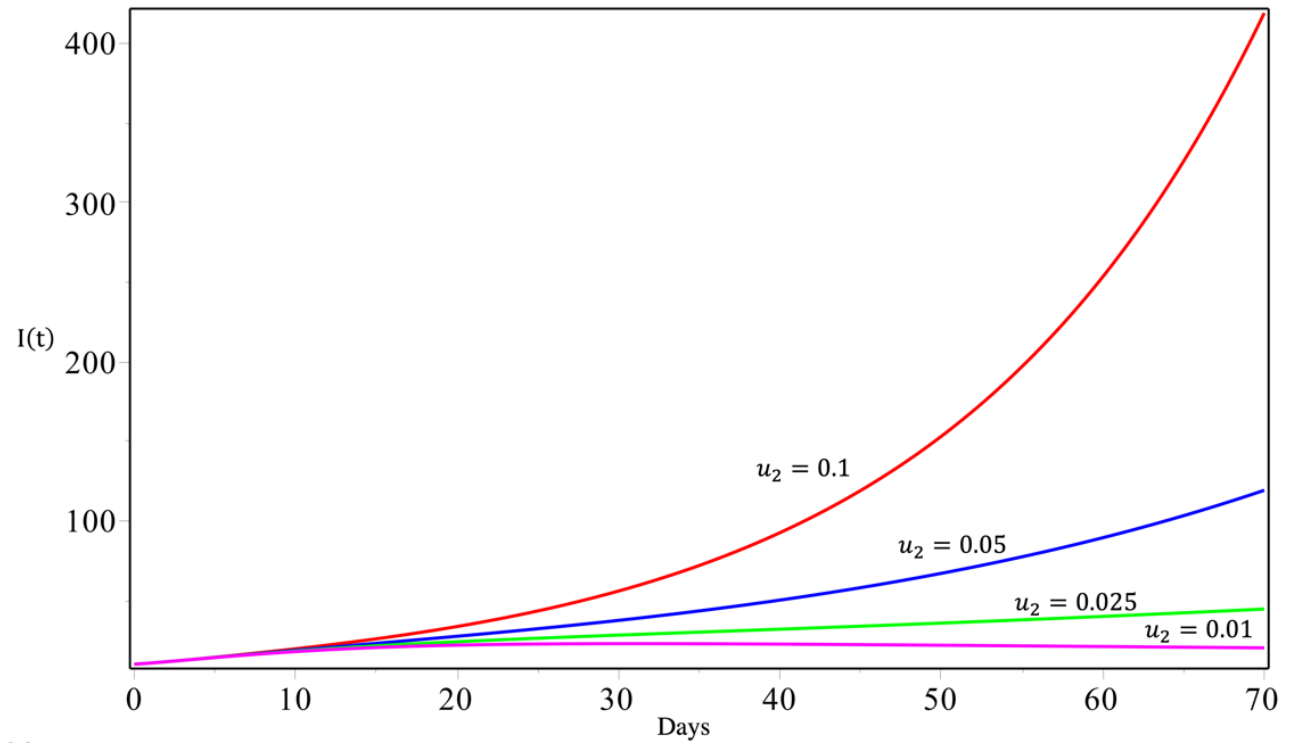

Fig. 4. Solution of trajectories of infected population of model (1) for various value of $u_{2}$.

\section{Conclusions}

In this paper, we formulate a system of the ordinary differential equation, which describes the transmission of COVID-19 among the human population. The model considers the direct transmission from the contact of human-to-human, and indirect transmission due to contact of human to a contaminated surface with the viruses. The model accounts for the media campaign effect to increase human awareness on the existence of COVID-19.

We show that system (1) always has a unique solution as long as the initial condition is positive. Furthermore, system (1) always has a unique COVID-19 free state, which is locally asymptotically stable (LAS) if the basic reproduction number is less than unity. We find that our model always has a unique COVID-19 endemic state whenever the basic reproduction number is larger than unity.

In the term of the sensitivity analysis of the basic reproduction number, we find that the media campaign succeeds to reduce the value of the basic reproduction number. We also find that we need a proper balance between the media campaign to increase human awareness and the media campaign to reduce the vanishment of people's awareness of the danger of COVID19 , such that we can increase the probability that COVID-19 could be eradicated from the population.

Although our model indicates the promising opportunity to rely on the media campaign for the COVID-19 eradication program, it also comes with a high cost for intervention. Hence, treat the media campaign parameter as a time-dependent variable could be a solution. The aim is to minimize the number of infected populations with minimum cost for the implementation program. This problem could be solved as an optimization problem. Please see [16-18, 21, 23-25] for some examples of optimization problems in epidemiological models. 


\section{Acknowledgements}

This research is financially supported by the Ministry of Research, Technology and Higher Education of the Republic of Indonesia (Kemenristek DIKTI) with the PUPT research grant scheme 2020 (ID Number: NKB-2803/UN2.RST/HKP.05.00/2020).

\section{References}

[1] Rothana HA, Byrareddy SN, The epidemiology and pathogenesis of coronavirus disease (COVID-19) outbreak, J. Autoimmun. (2020);109:102433.

[2] https://www.worldometers.info/coronavirus/. Accessed on 22 June, (2020).

[3] www3.nhk.or.jp. Accessed on April 17, (2020).

[4] Ferguson et al., Impact of non-pharmaceutical interventions (NPIs) to reduce COVID19 mortality and healthcare demand, Imperial College COVID-19 Response Team, (2020).

[5] D. Aldila et al., A mathematical study on the spread of COVID-19 considering social distancing and rapid assessment : The case of Jakarta, Indonesia, Accepted in Chaos, Solitons and Fractals, June (2020).

[6] D. Aldila et al., Optimal Control Problem Arising From COVID-19 Transmission Model With Rapid-Test, under review in Alexandria Engineering Journal, since June (2020).

[7] D. Aldila, Cost effectiveness analysis and backward bifurcation analysis on COVID-19 transmission model considering direct and indirect transmission, under review in Communications in Mathematical Biology and Neuroscience, since June (2020).

[8] Roosa, K., Lee, Y., Luo, R., Kirpich, A., Rothenberg, R., Hyman, J. M., Yan, P., and Chowell, G., Real-time forecasts of the COVID-19 epidemic in china from february 5 th to february 24th, 2020. Infectious Disease Modelling, 5:256 - 263, (2020).

[9] Abdelfatah Kouidere, Bouchaib Khajji, Amine El Bhih, Omar Balatif, Mostafa Rachik A mathematical modeling with optimal control strategy of transmission of COVID-19 pandemic virus Commun. Math. Biol. Neurosci., 2020 (2020), Article ID 24

[10] O. Torrealba-Rodriguez, R. A. Conde-Gutiérrez, A. L. Hernández-Javier, Modeling and prediction of COVID-19 in Mexico applying mathematical and computational models, Chaos, Solitons \& Fractals, Volume 138, September (2020), Article 109946

[11] Tingzhe Sun, Yan Wang, Modeling COVID-19 epidemic in Heilongjiang province, China Chaos, Solitons \& Fractals, Volume 138, September (2020), Article 109949.

[12] M. Highazy, Novel fractional order SIDARTHE mathematical model of COVID-19 pandemic Chaos, Solitons \& Fractals, Volume 138, September (2020), Article 110007.

[13] Sarbaz H. A. Khoshnaw, Muhammad Shahzad, Mehboob Ali, Faisal Sultan, A quantitative and qualitative analysis of the COVID-19 pandemic model Chaos, Solitons \& Fractals, Volume 138, September (2020), Article 109932.

[14] O. Diekmann, J. A. P. Heesterbeek, and M. G. Roberts, "The construction of nextgeneration matrices for compartmental epidemic models," Journal of the Royal Society Interface, vol. 7, no. 47, pp. 873-885, (2010).

[15] Aldila, D., Latifah, S. L., Dumbela P. A., Dynamical analysis of mathematical model for Bovine Tuberculosis among human and cattle population, Communication in Biomathematical Sciences, Vol. 2, No. 1., pp:55-64, (2019).

[16] Handari B.D., Vitra, F., Ahya R., Nadya S. T., Aldila D., Optimal control in a malaria model: intervention of fumigation and bed nets, Advances in Difference Equations, 2019:497 (2019), doi.org/10.1186/s13662-019-2424-6. 
[17] Bustamam, A., Aldila, D., Yuwanda, A., Understanding Dengue Control for Short- and Long-Term Intervention with a Mathematical Model Approach, Journal of Applied Mathematics 2018,9674138, (2018).

[18] Aldila, D., Handari, B.D., Widyah, A., Hartanti, G., Strategies of optimal control for hiv spreads prevention with health campaign, Communications in Mathematical Biology and Neuroscience 2020,7, (2020).

[19] Aldila, D., Seno, H., A Population Dynamics Model of Mosquito-Borne Disease Transmission, Focusing on Mosquitoes' Biased Distribution and Mosquito Repellent Use, Bulletin of Mathematical Biology 81(12), pp. 4977-5008, (2020).

[20] Aldila, D., Padma, H., Khotimah, K., Desjwiandra, B., Tasman, H., Analyzing the MERS disease control strategy through an optimal control problem, International Journal of Applied Mathematics and Computer Science 28(1), pp. 169-184, (2018).

[21] Aldila, D., Nuraini, N., Soewono, E., Optimal control problem in preventing of swine flu disease transmission, Applied Mathematical Sciences (69-72), pp. 3501-3512, (2014).

[22] N. Chitnis, J.M. Hyman, J.M. Cushing, Determining important parameters in the spread of malaria through the sensitivity analysis of a mathematical model, Bull. Math. Biol. 70 (5) (2008) 1272-1296.

[23] Rohman, M.I.S., Handari, B.D., Aldila, D., An impulse fumigation scenario to control dengue spreads, AIP Conference Proceedings 2023 (2018),020213.

[24] Hafidh, E.P., Aulida, N., Handari, B.D., Aldila, D., Optimal control problem from tuberculosis and multidrug resistant tuberculosis transmission model, AIP Conference Proceedings 2023 (2018),020223.

[25] Aldila, D., Nareswari, K., Tasman, H., An optimum control model for resistance fumigation for dengue, AIP Conference Proceedings 2021 (2018),060001. 